\section{Inflammatory debate}

The increased risk of tumour occurrence in patients with chronic inflammatory diseases has long been reported. NF- $\kappa \mathrm{B}$ is a transcription factor triggered in response to proinflammatory cytokines; a stimulatory role of NF- $\kappa B$ in cancer has been suspected, but not conclusively proven. Now two papers have directly addressed the effect of NF- $\kappa \mathrm{B}$ expression in tumorigenesis and describe related and intriguing findings.

Patients with the inflammatory bowel disease ulcerative colitis are treated with non-steroidal antiinflammatory drugs (NSAIDs) to reduce both disease symptoms and the increased risk of colitis-associated cancer (CAC). Michael Karin and colleagues investigated the activation of NF- $\kappa B$ in a mouse model of CAC. NSAIDs reportedly inhibit the function of an NF- $\kappa \mathrm{B}$ activator, IKK $\beta$. So, Karin and colleagues used mice with tissue-specific deletion of IKK $\beta$ to inhibit NF- $\kappa B$ activation.

To induce colonic tumours in these mice, the authors used a pro-carcinogen - azoxymethane - and three doses of the pro-inflammatory sodium salt dextran sulphate. Mice lacking IKK $\beta$ in their intestinal epithelial cells had a markedly reduced occurrence of tumours compared with controls, indicating that NF- $\kappa B$ is important for tumour initiation and/or promotion. What might be the underlying mechanism for this? The inflammatory response, which induced apoptosis in the epithelial cells, was more severe in mice with IKK $\beta$-deficient epithelial cells and was highlighted by the increased incidence of apoptosis in these cells. As NF- $\kappa \mathrm{B}$ is a potent antiapoptotic transcription factor, the authors concluded that the decreased rate of tumour incidence in these mice is due to increased apoptosis resulting from a lack of active NF- $\kappa B$.

But does NF- $\kappa$ B also contribute to tumorigenesis through the inflammatory response? To address this, the author's generated mice in which

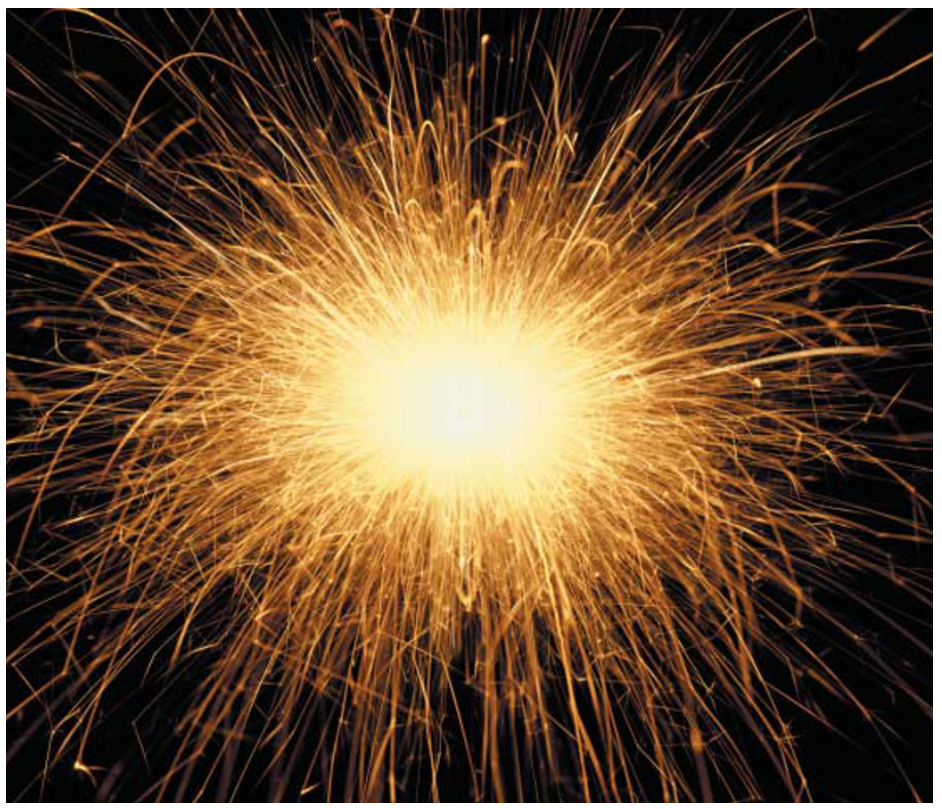

IKK $\beta$ was specifically absent in the myeloid/macrophage lineage and treated these mice with the same procarcinogen and pro-inflammatory regimen. Tumour incidence was reduced in mice with NF- $\kappa B$-deficient macrophages and correlated with lower levels of inflammatory cytokines, resulting in substantially reduced proliferation of intestinal epithelial cells. Therefore, the authors conclude that NF- $\kappa \mathrm{B}$ contributes to CAC through two mechanisms: through protecting intestinal epithelial cells against inflammationdriven apoptosis and by increasing epithelial-cell proliferation through the transcription of macrophage inflammatory cytokines.

Similar conclusions have been reached by Yinon Ben-Neriah and colleagues in their recent Nature paper. To investigate the contribution of NF- $\kappa \mathrm{B}$ to tumorigenesis they used multidrug resistance $2(M d r 2)$ knockout mice, which spontaneously develop cholestatic hepatitis, leading to hepatocellular carcinoma, a model of inflammatory-associated cancer. Initially, the authors showed that the inflammatory cytokine tumour-necrosis factor- $\alpha$ (TNF $\alpha)$ is produced by the invading inflammatory cells and surrounding endothelial cells, and results in increased NF- $\kappa \mathrm{B}$ expression in hepatocytes. To examine this further, the Mdr2-knockout mice were crossed with mice that have hepatocytes with regulatable NF- $\kappa \mathrm{B}$ activity. This showed that active NF$\kappa \mathrm{B}$ in hepatocytes was not required to drive the inflammatory process or for the evident hepatocyte proliferation and hyperploidy - characteristics of cholestatic hepatitis - or the early pre-malignant dysplastic state. However, analyses of older mice demonstrated that hepato cellular carcinoma size and incidence was reduced when NF- $\kappa \mathrm{B}$ was inactivated in dysplastic hepatocytes, correlating with a threefold increase in inflammatory-cytokine-induced apoptosis in these cells. also show that the expression of $\mathrm{NF}-\kappa \mathrm{B}$ contributes to tumorigenesis through suppression of apoptosis. Importantly, they also show that induction of apoptosis in dysplastic hepatocytes expressing NF- $\kappa \mathrm{B}$ can be achieved in vivo through the administration of TNF $\alpha$-neutralizing antibodies. Drugs that target TNF $\alpha$ are already used in clinical practice and the authors suggest that these might prove useful as chemopreventive agents in patients with chronic inflammatory diseases who are at an increased risk of developing cancer.

\section{(2) References and links} ORIGINAL RESEARCH PAPERS Greten, F. R. et al. IKK links inflammation and tumorigenesis in a mouse model of colitis-associated cancer. Cell 118, 285-296 (2004) | Pikarsky, E. et al. NF-kB functions as a tumour promoter in inflammationassociated cancer. Nature 25 Aug 2004 (doi:10.1038/nature02924)
So, Ben-Neriah and colleagues

Nicola McCarthy

\section{IN THE NEWS}

Data collecting dust

Decisions about cancer treatment are being hindered by the reluctance of some pharmaceutical companies to publish crucial clinical trial data or, in some cases, to even register their trials in the first place. Up to a quarter of cancer clinical trials are not published and are "being left to gather dust" (Independent, 08 Sept 2004), claimed Richard Sullivan, of Cancer Research UK, at the annual British Association Festival of Science.

Sullivan went on to explain, "The medical community needs to know the results of clinical trials to be able to view the entire picture of how a treatment works, how it compares to other therapies and what choices could serve patients best" (http://news.bbc.co.uk, 07 Sept 2004). Selective reporting further compounds the problem; by favouring positive results, investigators and journal editors could

"distort medical literature and leave doctors thinking a treatment is more effective than it actually is" (http://news.bbc.co.uk, 07 Sept 2004).

Moreover, as Fran Balkwill commented in a Cancer Research UK press release, "If the results from trials never reach the public domain researchers could waste time and unknowingly repeat lines of inquiry that have already been proven unsuccessful".

The problem is partly due to the disparity between public and private interests. Drug companies are disinclined to publish results that could place financial interests at risk, although these very results might help in the planning of cancer treatment.

Sullivan's comments are certainly timely, with an editorial in the New England Journal of Medicine (16 Sept 2004) also calling for all trials to be registered to facilitate full transparency with respect to clinical trial reporting.

Oliver Childs 